\title{
HISTÓRIA NATURAL E ESCRITA DE VIAGEM: A VOZ DE ELIZABETH CABOT CARY AGASSIZ'
}

\author{
NATURAL HISTORY AND TRAVEL NARRATIVE: \\ THE VOICE OF ELIZABETH CABOT CARY AGASSIZ
}

\section{Natália Fontes de Oliveira²}

RESUMO: A história natural e as narrativas de viagens, muito populares entre os séculos XVI e XIX, são marcadas pela exploração científica de culturas e territórios estrangeiros. Muitas vezes marginalizadas pela cultura impressa, viajar pelos mares concedia às mulheres a autoridade para serem detentoras da palavra. Elizabeth Cabot Cary Agassiz foi uma mulher pioneira que viajou ao Brasil com a Expedição Thayer ao lado do marido durantes os anos de 1865 a1866. Após um ano de retorno aos EUA, Elizabeth e o marido publicaram em coautoria a narrativa de viagem, $A$ Journey to Brazil. Apesar da publicação em conjunto, há uma falta de estudos que reconheçam a voz autoral de Elizabeth. Este artigo propõe uma análise da obra A Journey in Brazil para resgatar a contribuição de Elizabeth Cary Cabot Agassiz para a disseminação do conhecimento no século XIX. O referencial teórico, numa abordagem interdisciplinar, baseia-se em estudos sobre a escrita de viagens e a crítica literária feminista. Elizabeth dialoga com as principais vozes masculinas do século e com os discursos da história natural para criar um espaço literário único e inserir sua voz na cultura impressa.

PALAVRAS-CHAVE: Narrativas de viagem. Escrita de autoria feminina. História natural. Elizabeth Cabot Cary Agassiz.

ABSTRACT: Natural history and travel narratives, highly popular between the sixteenth and nineteenth centuries, is marked by scientific exploration of foreign cultures and territories. Although women were often marginalized from print culture, traveling across the seas granted them the authority to be the beholders of the pen. Elizabeth Cabot Cary Agassiz was a pioneer woman that traveled to Brazil with the Thayer Expedition alongside her husband from 1865 to 1866 . Upon a year with their return to the U.S. Elizabeth and her husband published in co-authorship the travel narrative, A Journey to Brazil. This article proposes an analysis of the travel narrative, A Journey in Brazil, to rescue Elizabeth Cary Cabot Agassiz's contribution to the dissemination of knowledge in the nineteenth century. Through an interdisciplinary approach, the theoretical framework is based on studies on travel writing and feminist literary criticism. Elizabeth dialogues with leading male voices of the century and natural history discourses to rewrite a unique literary space and insert her voice into print culture.

KEYWORDS: Travel narratives. Women's writing. Natural history. Elizabeth Cabot Agassiz.

${ }^{1}$ Artigo recebido em 30 de agosto e aceito em 10 de outubro de 2021.

${ }^{2}$ Professora do Departamento de Letras da Universidade Federal de Viçosa (UFV); Ph.D. em Literatura Comparada pela Purdue University; Coordenadora do Grupo de Pesquisa Mullheres e Ficção (UFV); membro do GT Mulher e Literatura da Anpoll. ORCID: https://orcid.org/0000-0003-0566-1791. E-mail: nataliafontes@ufv.br. 
A relação entre viagens, narrativas de viagem e o empreendimento da história natural é marcada por dinâmicas complexas e plurais. No século XIX, os avanços tecnológicos tornaram viagens pelos mares e publicações de escrita de viagem uma possível realidade para a sociedade burguesa. Mulheres se apropriaram dessa oportunidade para ganhar maior liberdade de mobilidade e autoria. Elizabeth Cabot Cary Agassiz (1822-1907) é uma pioneira americana que viajou ao Brasil com a Expedição Thayer (1865-66), ao lado de seu marido, Louis Agassiz (1807-1873). Com base em suas viagens, a narrativa de viagem, $A$ Journey is Brazil, é publicada em 1868 nos Estados Unidos. Embora a obra é assinada em coautoria, este artigo considera a Elizabeth como principal autora, referindo-se a mesma como Cabot-Agassiz. ${ }^{3} \mathrm{~A}$ autora cria uma linguagem própria, reescrevendo o espaço textual das narrativas de viagem. Este artigo propõe uma análise da obra A Journey in Brazil para resgatar a contribuição de Elizabeth Cary Cabot Agassiz para a disseminação do conhecimento no século XIX. Elizabeth dialoga com as principais vozes masculinas para reescrever um espaço literário único e inserir sua voz na cultura impressa.

Ao longo da história moderna, a participação de mulheres em práticas científicas, foi silenciada, especialmente o trabalho feito por mulheres viajantes. Em Viajantes Solitárias: Narrativas de Viagens e Vocação Científica de Mulheres no Século Dezenove, Lila Harper observa que a ciência nos tempos modernos se identifica com o sexo masculino (2001, p. 12). As vozes das mulheres foram marginalizadas do campo da ciência moderna e, autoria muitas vezes ignorada. As contribuições das mulheres para a ciência moderna foram minimizadas e mesmo ignoradas pelos pesquisadores:

Existia uma tendência de ignorar contribuições de mulheres que ajudavam suas famílias, frequentemente do marido, trabalhando como coletoras, catalogadoras e ilustradoras [...] estudos do século dezenove no contexto de uma ciência pré-profissional indicavam que mulheres estavam mais envolvidas com o desenvolvimento da história natural do que se suspeitava previamente (HAEPER, p. 12-13).

\footnotetext{
${ }^{3} \mathrm{~A}$ voz narrativa pertence à Elizabeth, uma vez que a voz Louis aparece no texto entre aspas e nos últimos capítulos claramente demarcados como escrito por ele com sua assinatura. Para maiores considerações a respeito de autoria da obra, ver Three Traveling Women Writers: Cross-Cultura Perspectives of Brazil, Patagonia and the U.S. de Natália Fontes de Oliveira.
} 
Harper fala diretamente sobre o papel fundamental das mulheres na construção do conhecimento científico como viajantes, escritoras e, colaboradoras de expedições científicas. MuIheres tiveram um grande papel na construção do conhecimento científico de história natural ao longo dos séculos, mas houve um o silênciamento dessas vozes de mulheres. Este artigo propõe uma viagem ao passado para enfatizar o papel ativo das mulheres no campo das ciências e das literaturas.

Elizabeth nasceu em uma das famílias mais tradicionais e influentes de Boston nos Estados Unidos, os Cabots. ${ }^{4}$ Os Cabots se preocupavam em cultivar as artes, apoiar instituições de caridade e assumir papel de liderança na sociedade. Elizabeth seguiu essa tradição defendendo a igualdade de educação das mulheres na comunidade de Harvard. Ela acreditava que as mulheres deveriam aprender as mesmas matérias e pelos mesmos professores que os homens. Em 1879, junto com Arthur Gilman e Alice Longfellow, Elizabeth lutou para criar o anexo de Harvard, que foi formalmente incorporado como a Society for the Collegiate Instruction of Women. Elizabeth foi a primeira presidente da Sociedade, e seu trabalho foi essencial para garantir que o Anexo de Harvard fosse declarado Radcliffe College em 1894. ${ }^{5}$ Apesar de ser reconhecida como membro da família Cabot, a voz literária de Elizabeth é menos conhecida. Nesse sentido, torna-se necessário esse resgate social cultural e literário para demonstrar a importância da voz da Elizabeth Cabot Cary Agassiz durante o século XIX. Para tal, analisamos como a autora dialoga com as principais vozes da época: Alexander von Humboldt e Carl Linneaus.

Alexander von Humboldt foi um dos mais influentes e discutido escritor de viagem na Europa e nas Américas do Norte e do

\footnotetext{
${ }^{4}$ Brahmin são compostas por famílias Yankee originarias da classe alta de Boston. O termo é importado da Índia, onde Brahim se refere à classe mais alta do sistema de castas. Nos Estados Unidos tem sido aplicado à classe alta de famílias da Nova Inglaterra descendentes de ingleses que eram influentes no desenvolvimento e liderança nas artes, ciência, política e academia. Membros da classe Brahmin formam uma parte significante da Costa Leste, comumente associada com a Universidade de Harvard. John Collins Bossidy escreveu a doggerel, ou cantiga, "Boston Toast", popular à época: "E esse é o velho e bom Boston// A casa do feijão e do bacalhau//Onde os LOwells conversam somente com os Cabots// And the Cabots conversam somente com Deus." Em inglês, no original: "'And this is good old Boston// The home of the bean and the cod// Where the Lowells talk only to Cabots// And the Cabots talk only to God."

${ }^{5}$ A obra Elizabeth Cabot Agassiz: A Biography de Lucy Allen Paton; e Women through Women's Eyes: Latin American Women in Nineteenth-Century Travel Accounts de June Hahner descrevem e analisam a vida da Elizabeth Cabot Agassiz.
} 
Sul $^{6}$. Nascido no mesmo ano de Napoleão, 1769, cada um tomou caminhos diferente para conquistar terras estrangeiras. Enquanto Napoleão estendeu o domínio francês através de guerras, Humboldt estendeu o domínio alemão através da escrita. $O$ autor influenciou a expansão econômica europeia ao idealizar a América do Sul para o consumo europeu. Em Imperial Eyes, Mary Louise Pratt sugere que: "a reinvenção da América foi um processo transatlântico que acionou energias e imaginações intelectuais do público de ambos hemisférios, mas não necessariamente da mesma forma" (2008, p. 110). Por meio das narrativas de viagens de Humboldt, as elites mundiais formaram suas opiniões sobre as Américas. Muitos exploradores e intelectuais, incluindo Charles Darwin, posteriormente se inspiraram em seu trabalho. Darwin escreveu que: "o caminho inteiro de sua vida decorre de ter lido e relido as Narrativas Pessoais de Humboldt na juventude" (BOTTING, 1973, p. 213). Humboldt estabeleceu uma tradição que consagrou a importância de escritas de viagem na formação das nações. Vindo de uma família abastada, ele não viajava como cientista com prazos e trabalhos a serem excutados, mas como um viajante independente, criando novos paradigmas a respeito de paisagens estrangeiras. Em Visões da Natureza, Humboldt descreveu os planaltos da Venezuela:

Aos pés de uma imponente faixa granítica, as quais nos anos iniciais do nosso planeta, resistiram às erupções das águas na formação do Golfo Caribenho, estende uma vasta e ilimitada planície [...] da rica luxúria da vida orgânica o atônito viajante de repente se encontra na margem sombria sem árvores. (HUMBOLDT, 1850, p. 3) ${ }^{7}$.

O uso de adjetivos como "ilimitada," "rica," "luxuria" exalta as riquezas da natureza para além da compreensão humana. Humboldt continua a pintar a natureza como grandiosa; associando a paisagem não como um panorama estático, mas como uma natureza viva:

\footnotetext{
${ }^{6}$ Humboldt tem sido tópico de muitos debates acadêmicos. Minha discussão a respeito das narrativas de Humboldt foi obtida de Hanno Beck em "The Geografy of de Alexander von Humboldt" em Wolfgang-Hagen Hein, ed., Alexander von Humboldt: Life and Work; Humboldt and the Cosmos de Douglas Botting, e Imperial Eyes de Mary Loiuse Pratt.

7 Todas as traduções literárias, exceto quando devidamente sinalizadas, são de minha autoria. A citação no original em inglês: "At the foot of the lofty granitic range which, in the early ages of our planet, resisted the irruption of the waters on the formation of the Caribbean Gulf, extends a vast and boundless plain [...] From the rich luxuriance of organic life the astonished traveler suddenly finds himself on the drear margin of a treeless waste".
} 


\begin{abstract}
A estreiteza desse extensivamente recuado continente na parte norte dos trópicos, onde a atmosfera repousa, ocasiona a subida de uma corrente de ar mais quente; sua larga extensão direcionada a ambos polos de gelo; o oceano varrido por ventos tropicais; a planicidade das margens do leste; correntes de águas geladas da região Antártica [...] e avança para o norte do Peru como Cape Parina, onde de repente divergem para o oeste; as numerosas montanhas na primavera [...] e o oceano com prodigiosa umidade, parcialmente absorvida e parcialmente gerada [...] nesses dependem a luxuria e exuberante vegetação e a riqueza das foIhagens que são peculiarmente características no Novo Continente (HUMBOLDT, 1850, p. 7-8) ${ }^{8}$.
\end{abstract}

Humboldt criou uma natureza viva com verbos como "repousa," "varrido," "avança," e "divergem", para realçar a natureza como um agente por si só, forte e poderosa. $O$ adjetivo exuberante e o substantivo riqueza reforçam o esplendor majestoso da beleza que se estende além do olhar humano. A natureza extrapola qualquer intervenção ou entendimento humano, sendo conectada a forças que ultrapassam o olhar humano. Pratt argumenta que a narrativa de Humboldt inspira a se pensar: "em uma câmera que continuamente se move e muda de foco - exceto que o visual na verdade não participa na descrição. Humboldt invoca um sistema da natureza não limitada visualmente, mas uma interminável expansão e contradição de forças invisíveis" (2008, p. 121). A metáfora de Pratt de uma câmera se encaixa bem com o estilo de narrativa de Humboldt, onde ele foca em uma natureza que se modifica de uma cena para a próxima.

A metáfora da câmara utilizada para descrever a narrativa de Humboldt, também pode ser empregada para analisar a descrição de Cabot-Agassiz em A Journey in Brazil. Como Humboldt, a autora se maravilhou com a imensidão da natureza da América

\footnotetext{
${ }^{8}$ No original em inglês: "The narrowness of this extensively indented continent in the northern part of the tropics, where the fluid basis on which the atmosphere rests, occasions the ascent of a less warm current of air; its wide extensions towards both the ice poles; a broad ocean swept by cool tropical winds; the flatness of the eastern shores; currents of cold seawater from the Antarctic region [...] and advance as far north on the coasts of Peru as Cape Parina, where they suddenly diverge toward the west; the numerous mountains abounding in springs [...] and the Ocean, prodigious quantities of moisture, partly absorbed and partly generated [...] on these alone depend the luxuriance and exuberant vegetation and that richness of foliage which are so peculiarly characteristic of the New Continent.
} 
do Sul. No entanto, se a natureza para Humboldt é mais do que os olhos podem ver, a natureza para Cabot-Agassiz se encaixa aos olhos humanos e está disponível para análises. Ela se desvia da majestosa e romântica natureza de Humboldt e sua escrita é marcada por um foco mais específico, detalhando as especificidades desse panorama. Evidencia-se esse olhar atento às descrições tangíveis, em sua narração sobre a chegada ao porto do Rio de Janeiro:

\begin{abstract}
A faixa costeira, embora não muito imponente (seu pico se encontra entre dois s três mil pés), é ousada e com muitos precipícios. Os picos são cônicos e as encostas íngremes se encontram á beira das águas, onde, em muitos lugares, uma larga praia aparece ao largo. $O$ pitoresco cenário se intensifica á medida que se aproxima a entrada da baía. Uma vez dentro desse estreito e rochoso portal, o imenso porto, avançando para o norte por mais de vinte milhas, parece mais com um vasto lago cercado por montanhas do que com uma baía. Em um lado se encontra os picos do Corcovado, a Tijuca, e a plana Gávea; no outro lado e mais em terra firme, as Montanhas Organ suspendem seus pontos enquanto dentro da entrada aparecem as pedras pretas conhecidas como Pão de Açúcar (CABOT-AGASSIZ, 1868, p. 46-47).
\end{abstract}

A descrição de Cabot-Agassiz parece possuir uma abordagem científica, uma vez que o cenário é detalhado com medidas e, evitando-se a linguagem romântica. Como Humboldt, ela caracterizou a natureza como um lugar amplo, utilizando construções como "imenso porto" e "vasto lago". Contudo, a sua visão é permitida aos olhares humanos. Os limites da natureza poderiam ser vistos, classificados e analisados. Para descrever o Corcovado, a linguagem de Cabot-Agassiz explora a imensidade da natureza de Humboldt ao mesmo tempo em que desafia tal tropo:

Um vasto prospecto panorâmico sempre ilude a descrição, mas certamente poucos combinam elementos raros de beleza como o cume do Corcovado. $\mathrm{O}$ enorme litoral com portas de entrada para o mar, o largo oceano à vista, as muitas ilhas, o círculo montanhoso com as nuvens sobrevoando os picos - todos esses recursos produzem um lindo cenário. Um 
grande charme desse cenário consiste no fato que, embora muito extensivo, não é tão distante que reprimem os objetos de sua individualidade. Afinal, uma visão muito distante é como um inventário: muitas florestas escuras; muitas paisagens verdes no campo; tantos pontos em branco, lagos; muitos rios, etc. (CABOT-AGASSIZ, 1868, p. 62).

Como Humboldt, que enfatizou uma natureza infinita e atemporal, Cabot-Assiz mencionou a imensidão de tais cenários, mas contextualizou o que estava vendo. Mesmo que ela mencione "os raros elementos de beleza", a aparência do Corcovado é abordada por meio de uma visão objetiva das montanhas e seus arredores. ${ }^{9}$ A associação da natureza com um inventário sugere o aspecto classificatório e a necessidade de organização dos diferentes elementos do cenário. A autora faz referências às nuvens, as muitas ilhas ao redor e, como o porto se abre para o mar, contextualizando a razão da visão ser tão linda sob um olhar objetivo. A natureza não era olhada em um vácuo, mas composta por diferentes níveis de flora. Em outro exemplo, ao invés de exclamar que a natureza do Rio era inexplicavelmente linda, ela tenta explicar por que o cenário era tão lindo:

As montanhas ao longo da estrada, bem como em todo o Rio, são de formas muito peculiares, íngreme e cônica, sugerindo à primeira vista uma origem vulcânica. É essa aspereza que proporciona grandiosidade às montanhas, uma média de altura que não ultrapassa dois ou três mil pés. Um exame mais detalhado da estrutura shows que seu fantástico formato é o resultado de um processo de desintegração, e não uma convulsão repentina (CABOT-AGASSIZ, 1868, p. 69) ${ }^{10}$.

\footnotetext{
${ }^{9}$ O Corcovado é uma montanha localizada na vizinhança da Tijuca na área central do Rio de Janeiro. Se encontra no topo da montanha do Cristo Redentor. Para mais discussões que representem a natureza das narrativas de viagens no Brasil, ver narrativas de Márcia Barbosa em "História Natural e Narrativas de Viagens: Novas relações, novas linguagens, novas imagens."

${ }^{10}$ No original em inglês: "The mountains along the road, as indeed throughout the neighborhood of Rio, are of very peculiar forms, steep and conical, suggesting at first sight a volcanic origin. It is this abruptness of outline which gives so much grandeur to mountain ranges here, the average height of which does not exceed two or three thousand feet. A closer examination of their structure shows that their wild, fantastic forms are the result of the slow process of disintegration, not of sudden convulsions.
} 
Ela desconstruiu uma beleza inalcançável de um cenário exótico para discutir a formação de montanhas, explicando a razão para sua aparência. Como era comum em suas descrições, Cabot-Agassiz usou medidas para qualificar o cenário, nesse caso "dois ou três mil pés" (169). A autora propôs um "exame mais detalhado," um método adotado por ela na narrativa de viagem para classificar e qualificar a natureza, fugindo dos preceitos românticos de Humboldt em direção à uma linguagem mais especifica característica da história natural.

Paradoxalmente, tanto a linguagem científica quanto a linguagem romântica estão presentes ao longo da narrativa de viagem. Embora Cabot-Agassiz defenda uma visão objetiva da natureza, ela também tem uma visão romântica da grandiosidade da natureza, que desafia sua análise objetiva. Um exemplo foi sua viagem pela Serra do Mar. Mesmo que o cenário a tenha maraviIhado, ela organiza e caracteriza o cenário:

À primeira vista das elevadas montanhas, o primeiro vislumbre do oceano, a visão da vegetação tropical são épocas da vida de alguém. Essa maravilhosa floresta Sul Americana se emaranha com parasitas gigantes de tal forma que parece mais com uma sólida e compacta massa verde do que tela frondosa, vibrando com toda brisa e transparente ao sol, que representa a zona temperada da floresta (CABOTAGASSIZ, 1868, p. 54) ${ }^{11}$.

A descrição de Cabot-Agassiz contém emoções e linguagem científica. Termos e adjetivos como "primeira vista," "primeiro vislumbre" e "maravilhosa," invocam a grandiosidade do cenário e as sensações experimentadas por um viajante inicial. A ênfase no vocábulo "primeiro/a" ressoa com os discursos românticos da época que perpetuavam a ideia de que viajantes Europeus são os primeiros a atestarem a beleza da natureza e conquistá-la. Embora ela se distancie dos tropos de Humboldt, existem momentos na narrativa de viagem onde ela invoca uma linguagem romântica e mais emoti-

\footnotetext{
${ }^{11}$ No original em inglês: The first view of high mountains, the first glimpse of the broad ocean, the first sight of tropical vegetation in all its fullness, are epochs in one's life. This wonderful South American forest is so matted together and intertwined with gigantic parasites that it seems more like a solid, compact mass of green than like the leafy screen, vibrating with every breeze and transparent to the sun, which represents the forest in the temperate zone.
} 
va. A visão de Humboldt das Américas se manifesta profundamente na imaginação de viajantes europeus e americanos do período. Vale ressaltar que Cabot-Agassiz é ciente das contribuições de Humboldt no campo de escritas de viagem porque ela o conhecia pessoalmente. Seu marido correspondia-se com Humboldt e ocasionalmente incluía seu nome nas aulas: "Os primeiros exploradores, no senso moderno, foi Humboldt no mundo físico" (CABOT-AGASSIZ, 1868, p. 7). Sua referência a Humboldt reforça sua ciência intelectual das tradições do gênero em escritas de viagem.

Cabot-Agassiz dialoga com a imensidade da natureza de Humboldt, mas geralmente foca em uma natureza mais accessível e passível de análise. Essa abordagem se encontra em sintonia com discurso usado por cientistas e pesquisadores de história natural, como o famoso Carl Linnaeus. Nesse sentido, a narrativa de viagem, A Journey in Brazil, também pode ser comparada com a tradição científica estabelecida pelo pioneiro naturalista, Carl Linnaeus, que se deteve em classificar e descrever sistemas da natureza. Em 1735, Linnaeus publicou The System of Nature e obteve grande sucesso assim como adeptos no meio científico. Como naturalista, Linnaeus, apresentou diversas contribuições no campo da história natural e a sistematização da ciência em geral. Ele incorporou a língua latina em suas classificações, alegando que todas as plantas na terra poderiam ser classificadas pelas características específicas em suas partes reprodutoras. Discutindo a contribuição de Linnaeus para a ciência e escritas de viagem, Pratt sugere que:

O projeto de sistematização de Linnaeus nunca foi popularizado antes, [...] viagens e escritas de viagem nunca seriam a mesma novamente. Na segunda metade do século dezoito, uma expedição sendo ou não primordialmente científica, ou o viajante um cientista, a história natural era uma parte (PRATT, 2008, p. 26).

Linnaeus incorpora história natural como essencial em narrativas de viagens, modelando a disseminação do conhecimento científico a partir do século dezoito. A extensão pela qual os escritores viajantes incorporaram a sistematização de Linnaeus sobre a natureza variam em função dos objetivos do viajante: Cabot-Assiz adaptou as classificações de Linnaeus sobre a natureza quando a mesma tentou caracterizar, organizar, coletar e transcre- 
ver nas narrativas de viagens as variadas espécies de fauna e flora encontradas no Brasil. Na narrativa A Journey to Brazil, há vários capítulos sobre viagens ao longo do Rio Amazonas. A autora classifica a flora ao longo dos bancos de areia do Rio Amazonas com um olhar científico:

Nós estamos tão próximos dos bancos de areia hoje, que podemos quase contar as folhas nas árvores, com uma excelente oportunidade para estudar os diferentes tipos de palmeiras. Em um primeiro momento o Assai estava mais conspícuo, mas agora aparecem muitos outros. O Miriti (Marutitia) é um dos mais bonitos, com pendentes aglomerações de frutas vermelhadas e espalhando suas enormes folhas de plumas cortadas como fitas [...] O Japatí (Rhaphia), com suas plumas de folhas, algumas vezes de quarenta a cinquenta pés em comprimento, parece, em consequência de sua haste curta, começar do chão (CABOT-AGASSIZ, 1868, p. 156) ${ }^{12}$

Cabot-Agassiz fornece detalhes particulares das diferentes frutas ao longo das margens. Depois do nome em português de cada fruta, há a tradução para o latim, a língua da ciência, de acordo com os preceitos da época. A autora mescla diferentes discursos uma vez que sua descrição dialoga com a linguagem científica enquanto também incorpora metáforas românticas como "folhas de plumas". Também acrescenta sua opinião pessoal: "um dos mais bonitos", evocando linguagem sentimental. Interessantemente, a autora evita usar termos exatos, característicos da linguagem científica. Ao contrário, adota os vocábulos "como" e "verme-Ihadas" que são comparações flexíveis, sujeitas ao olhar e sentimento do autor. A cor da fruta é avermelhada, lembra a cor vermelha, mas tem algo diferente ao mesmo tempo. Em outro exemplo, Cabot-Agassiz descreve sua saída de Manaus, observando a floresta vista ao longo do caminho:

\footnotetext{
${ }^{12} \mathrm{O}$ original, em inglês: "We are keeping so near to the banks today, that we can almost count the leaves on the trees, and have an excellent opportunity of studying the various kinds of palms. At first the Assai was most conspicuous, but now come in a number of others. The Mirití (Marutitia) is one of the most beautiful, with its pendant clusters of reddish fruit and its enormous, spreading, fan-like leaves cut into ribbons, one of which Wallace says is a load for a man. The Japatí (Rhaphia), with its plume-like leaves, sometimes from forty to fifty feet in length, seems, in consequence of its short stem, to start almost from the ground."
} 
Desde que deixamos Manaus a floresta tem sido menos luxuosa; é mais baixa no Solimões do que no Amazonas, mais esfarrapada e aberta. As palmeiras também são menos numerosas até agora, mas existe uma árvore aqui que as acompanham dignamente. Sua cúpula plana, arredondada, mas não cônica, se levanta acima da floresta, e, quando vista a distância, apresenta um caráter arquitetural, muito regular em sua forma. Essa majestosa árvore, chamada de Sumaumeira (Eriodendron Sumauma), é uma das poucas árvores nesse clima que troca suas folhas periodicamente, e agora levanta seu arredondado cume acima da verde massa de vegetação ao redor (CABOT-AGASSIZ, 1868, p. 198-199) ${ }^{13}$.

Sua escrita se assemelha á apropriação da natureza por Linnaeus, uma vez que descreve a floresta por uma aparente lente objetiva, atendo-se a nomenclatura Latina. Cabot-Agassiz utiliza vocabulário para pintar exatamente o que vê com detalhes, como "cúpula plana," "arredondada mas não cônica." A autora fornece o nome local e o nome latino da árvore, bem como comentários a respeito das características de tais árvores, enfatizando o aspecto científico de suas descrições. Com termos como "luxuosa," "dignamente," e "majestosa" ela adiciona um tom de grandiosidade em sua descrição e sentimental, destacando a natureza estrangeira e sua imensidão, ecoando as descrições comumente utilizados por Humboldt. Nesse sentido, Cabot-Agassiz utiliza-se de uma linguagem objetiva, comum aos discursos científicos da história natural no século dezenove, ao mesmo tempo em que adota uma linguagem emotiva, repleta de metáforas cotidianas para descrever a flora de maneira coloquial.

Cabot-Agassiz vai além da tradição de Linnaeus ao classificar suas observações da flora e fauna porque incorpora diferentes elementos em suas descrições, frequentemente fazendo referências à língua local e assim, dando espaço para o saber de comuni-

13 No original em inglês: "Since we left Manaos the forest has been less luxuriant; it is lower on the Solimoens than on the Amazons, more ragged and more open. The palms are also less numerous than hitherto, but there is a tree here which rivals them in dignity. Its flat dome, rounded but not conical, towers above the forest, and, when seen from a distance, has an almost architectural character, so regular is its form. This majestic tree, called the Sumaumeira (Eriodendron Sumau$\mathrm{ma}$ ), is one of the few trees in this climate which shed their leaves periodically, and now it lifts its broad rounded summit above the green mass of vegetation around it, quit bare of foliage". 
dades nativas do Brasil. Um exemplo é sua narração sobre peixes durante sua estadia no assentamento em vilas no Pará:

essa manhã Maia trouxe um soberbo Pirara (fish parrot). Esse peixe é bem conhecido da ciência; é um pesado e cabeçudo com um escudo ósseo sobre toda sua cabeça; geralmente sua cor é preta, mas possui um amarelo brilhante dos lados, tornando-se alaranjado aqui e ali. Seu nome sistemático é Phractcephalus bicolor" (CABOT-AGASSIZ, 1868, p. 183) $)^{14}$.

Nesse trecho, a autora demonstra seu conhecimento sobre o campo das ciências e o que está catalogado nos anais científicos. Cabot-Agassiz adota o nome científico em Latim da espécie de peixe, mas também incluí o termo local em português, Pirara, oferecendo em parênteses a tradução para o inglês. Dessa forma, Cabot-Agassiz gerencia a inclusão de diferentes elementos em sua narrativa de viagem ao dialogar com tradições literárias e científicas e, inovar ao inserir conhecimentos locais em paralelo com descrições científicas. Sua visão científica é mais ampla do que muitos naturalistas influenciados por Linnaeus, uma vez que inclui o saber local de indígenas e, assim fortalecendo a alteridade. A autora continua sua descrição: "A gordura amarela desse peixe tem uma curiosa propriedade: os índios nos informam que quando papagaios são por ele alimentados eles são tingidos de amarelo, e rendem seus papagaios com mais variedade" (CABOT-AGASSIZ, 1868 , p. 183). ${ }^{15}$ Indígenas são retratados como conhecedores de peixe e descrevem os animais de acordo com suas visões. Ao incluir como as pessoas veem e usam os peixes nessas terras, Cabot-Agassiz recusa utilizar apenas uma linguagem cientifica distante de qualquer reconhecimento de saberes locais. Como Linnaeus, ela pode ser considerada uma naturalista, uma vez que foca nas descrições sobre flora e fauna, mas a autora vai muito além dessa tradição. Ao discutir aspectos da natureza sob diferentes pontos

14 No original, em inglês: "This morning Maia brought in a superb Pirarara (fish parrot). This fish is already well known to science; it is a heavy, broad headed hornpout, with a bony shield over the whole head; its general color is jet black, but it has bright yellow sides, deepening into orange here and there. Its systematic name is Phractocephalus bicolor".

15 No original: "The yellow fat of this fish has a curious property; the Indians tell us that when parrots are fed upon it they become tinged with yellow, and they often use it to render their 'papagaios' more variegated". 
de vistas, Cabot-Agassiz transgride a tradição naturalista abrindo espaço na narrativa para diferentes conhecimentos, incluindo as práticas culturais de indígenas locais.

Sexualidade era um tabu no século XIX, principalmente para as mulheres. Quando Linneaus propôs uma classificação de plantas baseada na sexualidade de plantas, causou um reboliço entre o fim do século dezessete e século dezenove. O sistema de Linnaeus de caracterização da sexualidade das plantas se assemelha aos laços matrimoniais introduzindo termos como andria e gynia originários dos gregos, marido (aner) e mulher (gyne) (SCHIEBINGER, 1996, p. 167). Nesse sentido, a transgressão do assunto não ultrapassa paradigmas patriarcais, ao incorporar termos relativos à casamento para se falar sobre homem e mulher. A sexualidade das plantas virou sensação entre os naturalistas, mas para as mulheres o tema era considerado inapropriado e, até mesmo perigoso.

A discussão explicitamente detalhada sobre a sexualidade das plantas poderia reduzir a autoridade de Cabot-Agassiz como intelectual e escritora de respeito. Para evitar discriminação sobre tais discussões, a reprodução de plantas não é enfatizada na narrativa de viagem. No entanto, sua retórica utilizada na descrição das florestas do Rio de Janeiro, ressoa com a linguagem sexualizada usada para descrever a natureza: "A beleza da vegetação é destacada aqui pela característica singular do solo. A cor da terra é peculiar do Rio; com uma rica e quente tonalidade vermelha, parece brilhar abaixo das videiras e plantas acima, e de vez em quando emerge para fora em contraste com o verdume ao redor" (CABOT-AGASSIZ, 1868, p. 60). ${ }^{16} \mathrm{O}$ solo, em outras palavras, um terreno fértil, é descrito como uma linda "rica e quente tonalidade vermelha" escondido abaixo das massas de "videiras e plantas acima," o qual pode sugerir uma referência ao órgão sexual da mulher. A sugestão do movimento "esporadicamente nasce" alude a movimentos sexualizados e/ou exposição do corpo feminino, em contraste às árvores verdes ao redor, que também podem ser representadas por um símbolo fálico. Mesmo que a retórica não seja intencionada a ser erótica, a descrição da natureza ultrapassa

${ }^{16}$ No original em inglês: "The beauty of vegetation is enhanced here by the singular character of the soil. The color of the earth is peculiar all about Rio; of a rich warm red, it seems to glow beneath the mass of vines and large-leaved plants above it, and every now and then crops out in vivid, striking contrast to the surrounding verdure". 
a tradição naturalista sobre sexualidade das plantas para incorporar implicitamente uma linguagem com conotação sexual, evitando o olhar científico.

Mulheres e naturezas tem uma complexa relação de longa data. Representações da natureza como mulher é um tema central em escritas de viagem e discursos sobre história natural. Ludmilla Jordanova demonstra que "a identificação da mulher com a natureza, o país, materialidade, passividade e sentimento era simultaneamente significativo da falta" (1999, p. 6). Tal oposição binária classifica mulheres como submissas e passivas. Comum na narrativas de viagem, a terra se encontra á espera de homens europeus para ser conquistada e controlada. Tal discurso androcêntrico é amplamente aceito e presente em narrativas científicas e de ficção. Cabot-Agassiz evita esse olhar para com as terras do Brasil, mas a natureza ainda é representada como feminina. Enquanto em uma pequena cidade do Pará, escreve:

O esqueleto de uma casa se encontra a beira do caminho; se em ruína ou inacabada, não posso dizer, mas de todo modo somente as paredes estão de pé, com as aberturas para portas e janelas. A natureza completou sua habitação imperfeita, - ela a cobriu com um telhado verde, ela cercou o vazio com um jardim de sua própria escolha; ela treinou videiras em torno das portas e janelas abertas; e a casa deserta, se não possui moradores, se encontra ao menos como casa para os pássaros (CABOT-AGASSIZ, 1868, p. 141) ${ }^{17}$

A autora primeiro menciona as condições de ruína da casa, que independente da razão, está incompleta. Diferentemente a natureza está completa e cheia de força ao apropriar-se do cenário "de sua própria escolha". Em sintonia com paradigmas tradicionais de escritas de viagem e outros gêneros literários, a natureza é feminina. Contudo, essa feminilidade é uma fonte de empode-

\footnotetext{
${ }^{17}$ No original em inglês: "The skeleton of a house stands by the wayside; whether a ruin or unfinished, I am unable to say, but at all events only the walls are standing, with the openings for doors and windows. Nature has completed this imperfect dwelling; - she has covered it over with a green roof, she has planted the empty enclose with a garden of her own choosing; she has trained vines around the open doors and window; and the deserted house, if it has no other inmates, is at least a home for the birds".
} 
ramento. A natureza não é retratada como uma terra passiva esperando ser conquistada por um homem. Ela por si só assumiu o controle sobre a casa, expandindo as videiras ao longo de portas e janelas. A natureza se rebela contra a arquitetura e Cabot-Agassiz subverte a associação entre mulher e natureza como frágeis e dóceis, descrevendo uma natureza empoderada que domina a casa. Com base na associação da natureza com mulher da época, pode-se interpretar que, assim como a natureza, as mulheres rejeitam a cultura do patriarcado e lutam por uma autonomia autoral e cívica. A natureza de Cabot-Agassiz pode ter resquícios de uma natureza feminina comum a discursos de história natural, mas a autora sugere que se a natureza é feminizada, ela é empoderada.

Ao longo da história ocidental, mulheres foram comumente marginalizadas e suas contribuições excluídas de cânones literários e discussões científicas. Elizabeth Cabot Agassiz lutou contra esse silenciamento ao escrever e publicar a narrativa de viagem A Journey in Brazil. Este artigo resgata a contribuição de Cabot-Agassiz para a disseminação do conhecimento científico durante o século dezenove. A autora habita e desafia os limites do discurso da história natural, reescrevendo paradigmas patriarcais do gênero. Na narrativa de viagem, Elizabeth cria uma linguagem própria, reescrevendo o espaço textual das narrativas de viagem ao dialogar com as tradições estabelecidas por Alexander von Humboldt e Carl Linneaus. Com esta pesquisa, espera-se inspirar futuros trabalhos que busquem esse resgate historio social e literário das vozes de mulheres nas ciências e nas literaturas.

\section{Referências}

BECK, H. Alexander Von Humboldt: Life and Work. Berlin: Wolfgang Hagen Hein, 1987.

BIRKETT, D. Off the Beaten Track: Three Centuries of Women Travellers. London: National Portrait Gallery, 2004.

BOTTING, D. Humboldt and the Cosmos. New York: Harper\&Row, 1973.

CABOT-AGASSIZ, E.; AGASSIZ, L. A Journey in Brazil. Boston: Ticknor and Fields, 1868. 
FONTES DE OLIVEIRA, N. Three Traveling Women Writers: Cross-Cultural Perspectives of Brazil, Patagonia and the U.S. from the Nineteen Century. New York: Routledge, 2017.

HAHNER, J.E. (Org.). Women through Women's Eyes: Latin American Women in Nineteenth-Century Travel Accounts. Oxford: Roman \& Littlefield Publishers, 2005.

HARPER, L. Solitary Travelers: Nineteenth-Century Women's Travel Narratives and the Scientific Vocation. London: Rosemont Publishing, 2001.

HUMBOLDT, A. V. Views of Nature. Trans. E.C. Otte and Henry G. Bohn. London: Henry G. Bohn, 1850.

JORDANOVA, L. J. Natural Facts: A Historical Perspective on Science and Sexuality. In: SAMSON, C. Health Studies: A Critical and Cross-Cultural Reader. Oxford: Blackwell, 1999, p. 36-49.

LINNEAUS, C. Systema Naturae. vol.1. Lockington, Allen \&Co.: London, 1906.

PATON, L. A. Elizabeth Cary Agassiz: A Biography. Boston, Houghton Mifflin Company, 1919.

PRATT, M. L. Imperial Eyes: Travel Writing and Transculturation. $2^{\text {nd }}$ ed. New York: Routledge, 2008.

SCHIEBINGER, L. Gender and Natural History. In JARDINE, N.; SPARY, E.; SECORD, J. (Orgs.). The Cultures of Natural History. Cambridge: Cambridge University Press, 1996. p. 163-177. 